\title{
Excision dynamics of Vibrio pathogenicity island-2 from Vibrio cholerae: role of a recombination directionality factor VefA
}

\author{
Salvador Almagro-Moreno ${ }^{1,2}$, Michael G Napolitano' ${ }^{1}$ E Fidelma Boyd ${ }^{1 *}$
}

\begin{abstract}
Background: Vibrio Pathogenicity Island-2 (VPI-2) is a $57 \mathrm{~kb}$ region present in choleragenic $\mathrm{V}$. cholerae isolates that is required for growth on sialic acid as a sole carbon source. V. cholerae non-O1/O139 pathogenic strains also contain VPI-2, which in addition to sialic acid catabolism genes also encodes a type 3 secretion system in these strains. VPI-2 integrates into chromosome 1 at a tRNA-serine site and encodes an integrase intV2 (VC1758) that belongs to the tyrosine recombinase family. IntV2 is required for VPI-2 excision from chromosome 1, which occurs at very low levels, and formation of a non-replicative circular intermediate.

Results: We determined the conditions and the factors that affect excision of VPI-2 in V. cholerae N16961. We demonstrate that excision from chromosome 1 is induced at low temperature and after sublethal UV-light irradiation treatment. In addition, after UV-light irradiation compared to untreated cells, cells showed increased expression of three genes, intV2 (VC1758), and two putative recombination directionality factors (RDFs), vefA (VC1785) and vefB (VC1809) encoded within VPI-2. We demonstrate that along with IntV2, the RDF VefA is essential for excision. We constructed a knockout mutant of vefA in V. cholerae N16961, and found that no excision of VPI-2 occurred, indicating that a functional vefA gene is required for excision. Deletion of the second RDF encoded by vefB did not result in a loss of excision. Among Vibrio species in the genome database, we identified 27 putative RDFs within regions that also encoded IntV2 homologues. Within each species the RDFs and their cognate IntV2 proteins were associated with different island regions suggesting that this pairing is widespread.

Conclusions: We demonstrate that excision of VPI-2 is induced under some environmental stress conditions and we show for the first time that an RDF encoded within a pathogenicity island in $V$. cholerae is required for excision of the region.
\end{abstract}

\section{Background}

Vibrio cholerae is the etiological agent of the severe diarrheal disease cholera. Similar to many Gram-negative enteric pathogens, horizontal gene transfer and recombination plays a significant role in the evolution and emergence of new pathogenic strain of this species [1-12]. The main cause of the explosive rice water diarrhea characteristic of cholera is the cholera toxin $(\mathrm{CT})$, an $A B$ type enterotoxin, which is encoded within the ssDNA filamentous phage CTX $\phi[13,14]$. The B subunit of $\mathrm{CT}$ binds to the $\mathrm{GM}_{1}$ gangliosides, which are exposed

\footnotetext{
* Correspondence: fboyd@udel.edu

'Department of Biological Sciences, University of Delaware, Newark, DE 19716 USA

Full list of author information is available at the end of the article
}

when higher order gangliosides found in the intestinal mucus are cleaved by sialidase/neuraminidase $(\mathrm{NanH})$. This protein is encoded within a $57 \mathrm{~kb}$ region named Vibrio Pathogenicity Island-2 (VPI-2) [15,16]. In addition to encoding sialidase, VPI-2 also encodes the sialic acid catabolism (SAC) gene cluster (Figure 1A) [16-19]. The SAC cluster was shown to be present only in pathogenic isolates of $V$. cholerae and enables the bacterium to grow on sialic acid as a sole carbon source $[18,20]$. Recently, we demonstrated that the ability to catabolize sialic acid gives $V$. cholerae a competitive advantage in vivo [19]. In non-O1/O139 pathogenic isolates, in addition to the SAC cluster are the genes required for a type 3 secretion system which is important for virulence [21-25]. The toxin co-regulated pilus

\section{Biomed Central}




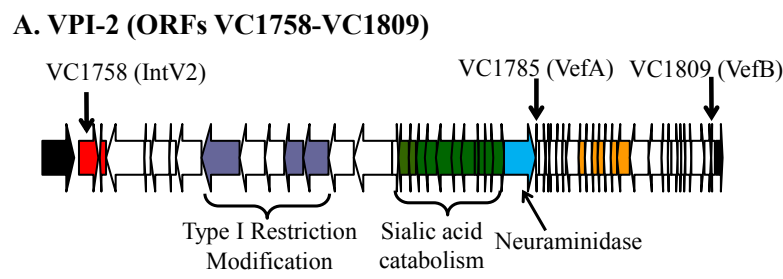

B. VPI-2 excision

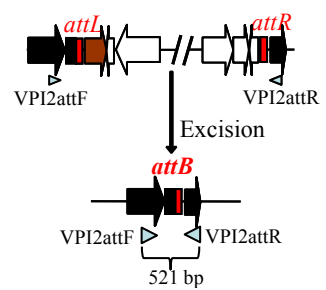

Figure 1 Vibrio Pathogenicity Island-2 (VPI-2) ORFs and primers used in this study. A. Schematic representation of VPI-2. Small black vertical arrows mark ORFs VC1758 (IntV2), VC1785 (VefA), or VC1809 (VefB). Block arrows represent ORFs and direction of transcription. Black arrows represent core genome ORFs (VC1757 and V(1810) present in all V. cholerae isolates; red arrow indicates an integrase, purple arrows indicate type 1 restriction modification ORFs, green arrows represent sialic acid catabolism, transport and regulator ORFs, blue arrow indicates sialidase/neuraminidase and yellow arrows represent transposase and Mu-phage like proteins. B. Schematic of VPI-2 excision mechanism and primer pair VPI2attF and VPI2attR used to detect the VPI-2 attB locus after excision of the entire region.

(TCP), an essential intestinal colonization factor for $V$. cholerae, is encoded within the $40 \mathrm{~kb}$ Vibrio Pathogenicity Island-1 (VPI-1 or TCP Island) region [26,27].

VPI-1 and VPI-2 do not share any genes in common but do share some functional characteristics such as the ability to integrate into the chromosome, specifically at a tRNA site using an integrase belonging to the tyrosine recombinase family $[16,18,23,26,28]$. VPI-2 integrates into chromosome 1 at a tRNA-serine locus, whereas VPI-1 is located at the tmRNA locus. Both regions are flanked by direct repeats (DRs) named attL and attR $[16,18,23,26,28]$. These integrases, IntV1 (VC0847) and IntV2 (VC1758), are believed to mediate insertion into the host chromosome through site specific recombination between an attachment site attP, present in the pathogenicity island, and $a t t B$, present in the bacterial chromosome.

Pathogenicity islands have been shown to excise from their host genome in pathogenic Escherichia coli and Yersinia species [29-36]. In E. coli strain 536, a uropathogenic isolate, Hacker and colleagues have identified six PAIs, all of which encode a tyrosine recombinase integrase and are flanked by DRs [31,33,36-39]. They demonstrated that PAI-I, II, III and V can excise from the chromosome by site-specific recombination involving their respective DRs (attL and attR) [31,33]. The PAIs were shown to excise at different frequencies depending on the growth conditions [31,33]. Likewise, both VPI-1 and VPI-2 have been shown to excise from their host chromosome [23,28]. Rajanna and colleagues demonstrated that VPI-1 can excise from $V$. cholerae N16961 at very low rates [28]. They determined that the integrase IntV1 (VC0847) was not essential for excision since a transposase within the region appeared to compensate for an IntV1 knockout [28]. Recently, Murphy and Boyd demonstrated that VPI-2 from $V$. cholerae N16961 can excise from chromosome 1, which also occurred at very low frequency under optimal growth conditions [23]. Their study showed that IntV2 (VC1758) was essential for excision and the formation of a circular intermediate (CI) [23]. Pathogenicity islands from both $E$. coli and $V$. cholerae are non-self mobilizable, they do not encode any proteins such as those for phage structural proteins or conjugation systems needed for cell to cell mobility $[23,28,31,33,36-39]$. The mechanism of transfer for most pathogenicity islands remains to be elucidated but likely involves hitchhiking with plasmids, conjugative transposons, Integrative and Conjugative Elements (ICEs), or generalized transducing phages or uptake by transformation.

It is known that for some mobile and integrative genetic elements (MIGEs) the presence of a recombination directionality factor (RDF)/excisionase is required for excision [40,41]. For instance, Xis is required for the excision of the ICE SXT from $V$. cholerae [41], Hef from the High Pathogenicity Island of Yersinia pestis [32], and Rox from the Shigella Resistance Locus (SRL) of Shigella flexneri [42]. RDFs are small basic proteins that bind and bend DNA on the recombination sites att $L$ and $a t t R$ triggering excision by coordinating the assembly of the excisive intasome [43-45]. In addition, some RDFs have been found to inhibit reintegration of the CI by converting attP into a catalytically inactive structure and are thought to stabilize the appropriate positioning of the integrase within the excisive intasome [46-48]. To date, no RDFs have been identified in $E$. coli or $V$. cholerae pathogenicity islands.

Here, we report the environmental conditions that induce excision of VPI-2. We examined the VPI-2encoded factors that are required for VPI-2 excision, determining that $V$. cholerae cells subjected to stress conditions showed an increase in the excision levels of VPI-2 compared to cell grown at optimal conditions. Bioinformatic analysis of the VPI-2 region identified two open reading frames (ORFs) VC1785 and VC1809 that show homology to previously described RDFs, which we named VefA and VefB. We examined the role of these genes in VPI-2 excision. 
Table 1 Bacterial strains and plasmids used in this study.

\begin{tabular}{|c|c|c|}
\hline Strains/plasmids & Genotype and/or phenotype & Reference \\
\hline \multicolumn{3}{|l|}{ V. cholerae } \\
\hline N16961 & O1 El Tor, VPI-2 +, Sm & {$[57]$} \\
\hline RAM-1 & $\mathrm{N} 16961, \Delta \mathrm{V} C 1758, \mathrm{Sm}^{\mathrm{R}}$ & [23] \\
\hline SAM-1 & RAM-1, plntV2, $S m^{R} \mathrm{Cm}^{R}$ & This study \\
\hline SAM-3 & $\mathrm{N} 16961, \Delta \mathrm{VC} 1785, \mathrm{Sm}^{\mathrm{R}}$ & This study \\
\hline SAM-4 & N16961, $\Delta$ VC1809, Sm ${ }^{R}$ & This study \\
\hline SAM-5 & SAM3, pVefA, Sm ${ }^{R} \mathrm{Cm}^{R}$ & This study \\
\hline SAM-11 & N16961, pBAD33, $\mathrm{Sm}^{R} \mathrm{Cm}^{R}$ & This study \\
\hline SAM-12 & RAM-1, pBAD33, $\mathrm{Sm}^{\mathrm{R}} \mathrm{Cm}^{\mathrm{R}}$ & This study \\
\hline SAM-13 & SAM-3, pBAD33, $\mathrm{Sm}^{\mathrm{R}} \mathrm{Cm}^{\mathrm{R}}$ & This study \\
\hline \multicolumn{3}{|l|}{ Plasmids } \\
\hline pDS132 & Suicide plasmid, $\mathrm{Cm}^{\mathrm{R}}$, SacB & {$[59]$} \\
\hline pBAD33 & Expression plasmid, Ara, $\mathrm{Cm}^{\mathrm{R}}$ & {$[60]$} \\
\hline plntV2 & vc1758 cloned into pBAD33 & This study \\
\hline pD1785 & $\Delta \mathrm{VC1785}$ cloned into pDS132 & This study \\
\hline pD1809 & $\Delta$ VC1809 cloned into pDS132 & This study \\
\hline pVefA & vc1785 cloned into pBAD33 & This study \\
\hline
\end{tabular}

\section{Methods}

\section{Bacterial strains and growth conditions}

The strains and plasmids used in this study are listed in table 1. Bacteria were grown in lysogeny broth more commonly known as Luria-Bertani broth (LB), LB agar, or LB agar $10 \%$ sucrose without $\mathrm{NaCl}$ (LB-Suc) [49]. Strains harboring the pBAD33 expression vector were grown on LB supplemented with $0.02 \% \mathrm{~W} / \mathrm{V}$ of L-Arabinose (LB-Ara). Bacteria were incubated overnight at $37^{\circ} \mathrm{C}$ with aeration unless otherwise indicated. When required, ampicillin (Amp, $100 \mu \mathrm{g} / \mathrm{ml}$ ), streptomycin $(\mathrm{Sm}, 200 \mu \mathrm{g} / \mathrm{ml})$, or chloramphenicol $(\mathrm{Cm}$, $25 \mu \mathrm{g} / \mathrm{ml}$ ) were added to the media.

\section{Determination of VPI-2 excision rate}

Excised circular VPI-2 DNA containing attP is expected to be a very rare event given the predicted low excision rate under normal conditions and the inability of VPI-2 to replicate after excision [23]. Therefore, we quantified the excision rates of VPI- 2 by measuring the presence of $a t t B$, the locus present on the $V$. cholerae chromosome after VPI-2 excises (Figure $1 \mathrm{~B}$ ), in different strains under different conditions, and comparing it with the presence of $a t t B$ in cultures of $V$. cholerae N16961 grown under standard optimal conditions: 12 hours in $\mathrm{LB}$ at $37^{\circ} \mathrm{C}$ with aeration. Using the O.D. values of $1 \mathrm{~mL}$ of a culture of $V$. cholerae $\mathrm{N} 16961$ grown for 12 hours in $\mathrm{LB}$ at $37^{\circ} \mathrm{C}$ with aeration as a reference, $750 \mu \mathrm{L}$ to $4 \mathrm{~mL}$ were pelleted by centrifugation and genomic DNA was extracted using ABI PrepMan Ultra reagent from the test cultures. We took $50 \mu \mathrm{L}$ from each DNA extraction and diluted each with $200 \mu \mathrm{L}$ of sterile $\mathrm{ddH}_{2} \mathrm{O}$. A $5 \mu \mathrm{L}$ aliquot of DNA after dilution was used as template for Real-Time quantitative PCR (QPCR) reactions. The QPCR assay calculated the percentage of cells in a culture that contained an unoccupied VPI-2 attB site. We quantified $a t t B$ sites present in cell grown under different growth conditions and normalized to the amount of attB present in N16961 grown for 12 hours at $37^{\circ} \mathrm{C}$. The gene-specific primers were designed using Primer3 software according to the real-time PCR guidelines, and are listed in Table 2. The Applied Biosystems 7000 system was used for RT fluorescence detection of PCR products that resulted from binding of the dye SYBR Green to double stranded DNA and the results were examined with Applied Biosystems SDS software $\mathrm{V}$ 1.3. The reference gene $m d h$ was assayed both separately and in the same reaction. To confirm that primer pairs only amplified target genes to assure accurate quantification of the results, non-template controls were included in each replicate. The $a t t B$ and $m d h$ PCR products were visually checked on agarose gels. The melting curves of PCR products were used to ensure the absence of primer dimers, contamination with genomic DNA and non-specific homologous sequences. PCR reactions were performed in $10 \mathrm{uL}$ volumes containing $5 \mathrm{uL}$ of 2 X SYBR Green PCR Master Mix (Applied Biosystems), $900 \mathrm{~nm}$ of each primer, and $1 \mathrm{uL}$ of DNA template. PCR cycling conditions were $30 \mathrm{sec}$ at $95^{\circ} \mathrm{C}$ followed by 40 cycles of $15 \mathrm{sec}$ at $95^{\circ} \mathrm{C}$ and $30 \mathrm{sec}$ at $60^{\circ} \mathrm{C}$. Serial doubling dilutions were used as templates for QPCR to generate standard curves for each $\mathrm{PCR}$ reaction by plotting relative DNA concentrations versus $\log \left(C_{t}\right)$ value $\left(C_{t}\right.$ is the PCR cycle at which fluorescence rises beyond background). The $C_{t}$ value for $m d h$ was 15 cycles and for attB 30 cycles. Every sample was assayed in triplicate and each experiment was performed using a minimum of three different samples. Differences in the $a t t B$ ratio were extrapolated using the delta-delta $\mathrm{Ct}$ method as developed by Pfaffl [50].

\section{Bioinformatic analysis}

BLAST search was performed using Xis (ABA87014), an RDF from V.cholerae SXT ICE element required for excision, AlpA, a well known RDF from E. coli (AAA18418) and the Hef protein (NP_405464) from $Y$. pestis pathogenicity island, as seeds on the genome sequence of $V$. cholerae N16961 [51]. DNA sequences from putative RDFs were downloaded from GenBank and the sequences were aligned using ClustalW [52]. Next, the protein sequences of characterized RDFs were used as seeds to perform BLASTN and BLASTP searches against Vibrio genomes sequences in the database [51]. The retrieved sequence must give an e-value below $10^{-3}$, relative to known RDFs. 
Table 2 Oligonucleotide primers used in this study.

\begin{tabular}{|c|c|c|}
\hline Oligo name & Sequence $\left(5^{\prime}-3^{\prime}\right)$. Restriction site underscored & Target gene \\
\hline \multicolumn{3}{|l|}{ SOE PCR } \\
\hline VC1785A & GAGCTCAATGGTGCATCGGCATATTCT & vefA (A-D) \\
\hline VC1785B & CAGCGATGATGGCGTGATTA & \\
\hline VC1785C & TAATCACGCCATCATCGCTGGGATGT TCTCCTATGTCTTGT & \\
\hline VC1785D & TCTAGACGCGCACATAACGCTGTTC & \\
\hline VC1809A & $\underline{\text { CTGCAGTGAGAGCAAGGGAAGTGATCGT }}$ & $\operatorname{vefB}(A-D)$ \\
\hline VC1809B & CGAGAGCTGGTTIACTTGTGTG & \\
\hline VC1809C & CACACAAGTAAACCAGCTCTCGGTGCATGATTGTCAAGTCATGCA & \\
\hline VC1809D & GAGCTCGATGGCTATGAATTAGCTCAGGA & \\
\hline \multicolumn{3}{|l|}{ Flanking } \\
\hline VC1785FF & GATGCTTCTATTACTCGGTT & vefA $(\mathrm{F}-\mathrm{R})$ \\
\hline VC1785FR & TCACCGCTGCTGCGTTAA & \\
\hline VC1809FF & GATTGATAGTAACAACACGCG & $\operatorname{vefB}(\mathrm{F}-\mathrm{R})$ \\
\hline VC1809FR & GTAATGCGCTATTGCTAAGTG & \\
\hline VPI2attF & AGAGTGAAAGTCGCCAAAGC & $a t t B(F-R)$ \\
\hline VPI2attR & GGGTGCAATTTCGCATGTTGC & \\
\hline \multicolumn{3}{|l|}{ Complements } \\
\hline VC1758CF & $\underline{\text { GAGCTCGAGTCCTCATGCTCTAGCCAG }}$ & intV2 (F-R) \\
\hline VC1758CR & TCTAGAGGCATGCTGGTGTGTTACTAC & \\
\hline VC1785CF & GAGCTCGCTTTGAATATAGGTAAGGGACTG & $\operatorname{vefA}(\mathrm{F}-\mathrm{R})$ \\
\hline VC1785CR & TCTAGACTATAGTACATGACGCATGTATAATC & \\
\hline \multicolumn{3}{|l|}{ Real-Time PCR } \\
\hline VPI2attBQF & GATTCGGTGAGTTGTCCGAGT & attB (VPI-2) (F-R) \\
\hline VPI2attBQR & GTGTTGGTGCAATGCTCAGTC & \\
\hline VC1758QF & TGCATGATCTTATACTCACCG & intV2 (F-R) \\
\hline VC1758QR & ACGTGCTCGCGGTTCATCTTC & \\
\hline VC1785QF & CCGCACTAAGCCGTTCAGCAA & $\operatorname{vefA}(\mathrm{F}-\mathrm{R})$ \\
\hline VC1785QR & CCATCCACTCATCCACTTCGC & \\
\hline VC1809QF & CTGAGAGGTGTGAATATGCCAG & $v e f B(F-R)$ \\
\hline VC1809QR & GTGATCCGTTGAGCAATCCAC & \\
\hline VC0432QF & TGTATGATATTGCGCCTGTCACAC & $m d h(F-R)$ \\
\hline VC0432QR & CCAGAACCACATCCGCACCTTC & \\
\hline
\end{tabular}

Underlined sequence represents restriction enzyme sites Sacl, Xbal and Pst1.

RNA extraction and Real-Time quantitative PCR (QPCR)

Total RNA from $V$. cholerae N16961 was extracted 12 hours post-inoculation in LB broth from one group treated with sub-lethal UV-irradiation and one group untreated as follows. The cells from $5 \mathrm{mLs} 11$ hours growth cultures were pelleted and resuspended in $5 \mathrm{mLs}$ of PBS. A $100 \mathrm{uL}$ aliquot was taken from each sample prior to treatment to calculate colony forming units (CFUs). Each $5 \mathrm{~mL}$ sample was placed in a plastic Petri dishes without a cover and one set of samples was irradiated with a sublethal dose of $25 \mathrm{~J} / \mathrm{m}^{2}$ of UV irradiation in a Fisher Scientific UV cross linker (FB-UVXL-1000) and the other $5 \mathrm{~mL}$ set of samples was left untreated as previously described by others [53]. The cells from both UV treated and untreated samples were recovered, pelleted, resuspended in $5 \mathrm{mLs}$ of LB broth and grown for 1 hour at $37^{\circ} \mathrm{C}$. A $100 \mathrm{uL}$ aliquot was taken from each sample to calculate CFUs post treatment from both sets of samples. The CFU counts pre and post treatment were identical at $\sim 9.75 \times 10^{9} / \mathrm{ml}$ as expected. Every experiment was performed in triplicate. Total RNA was extracted from each culture using RNAprotect Bacteria reagent (Qiagen, Valencia, $C A$ ) and an RNeasy mini kit (Qiagen) according to the manufacturer's protocols. RNA purity and the presence of genomic DNA were assessed using an ND-1000 NanoDrop UV-Vis spectrophotometer (NanoDrop Technologies) giving values of $A_{260} / A_{280}>2.0$ and $A_{260} / A_{230}>2.0$ indicating of no protein and solvent contamination, respectively. In addition, $1 \mu \mathrm{g}$ of each sample of RNA was run on a $1 \%$ agarose gel in $1 \times$ TBE buffer to examine quality of the samples. RNA was measured to calculate the volume of sample to be added to perform a reverse transcriptase (RT) reaction using SuperScript II Reverse Transcriptase 
and random hexamers following manufacturer's instructions (Invitrogen). The purity and quantity of cDNA was examined using an ND-1000 NanoDrop UV-Vis spectrophotometer as above. QPCR was performed using standard protocol using primer pairs for $v c 1758$, $v c 1785, v c 1809$ and $v c 0432$ (intV2, vefA, vefB and $m d h$, respectively) listed in Table 2 using SYBR green PCR Master Mix (Invitrogen) on an Applied Biosystems 7000 Real Time PCR System (Foster City, CA). To confirm that primer pairs only amplified target genes to assure accurate quantification of the results, non-template controls were included in each replicate. The int $V 2$, vef $A$, $v e f B$ and $m d h$ PCR products were visually checked on agarose gels. The melting curves of PCR products were used to ensure the absence of primer dimers, contamination with genomic DNA and non-specific homologous sequences. The data was analyzed using ABI PRISM 7000 SDS software (Applied Biosystems). Differences in the gene ratios were extrapolated using the delta-delta $\mathrm{Ct}$ method [50]. Every sample was assayed in triplicate and each experiment was performed using a minimum of three different samples.

\section{Construction of mutant strains}

To construct the mutant strains, primers were designed to conduct Splice Overlap Extension (SOE) PCR followed by allelic exchange [54]. SOE PCR primers were designed to produce non-functioning constructs of the 204-bp vef $A$ and the 228-bp vefB genes. The size of the regions removed from vef $A$ and $v e f B$ is 169 -bp and 191-bp, respectively and were constructed in $V$. cholerae strain N16961 to create mutant strains $V$. cholerae SAM-3 and SAM-4, respectively (Table 1). Primer pairs SOEVC1785A/SOEVC1785B and SOEVC1785C/ SOEVC1785 D were used to amplify PCR products from VC1785 from $V$. cholerae strain N16961 (Table 2). The ligated product was amplified with primer pair SOEVC1785A and SOEVC1785 D, which was restricted with enzymes, $\mathrm{XbaI}$ and SacI and ligated with pDS132 (New England Biolabs) resulting in $\mathrm{p} \Delta 1785$. p $\Delta 1785$ was transformed into $E$. coli strain DH5 $\alpha \lambda$ pir, plasmid purified and then transformed into $E$. coli $\beta 2155$ cells. E. coli $\beta 2155$ transformants were conjugated with N16961. V. cholerae cells were passaged in LB-suc to cure them of the integrated $\mathrm{p} \Delta 1785$. PCR was used to screen for $V$. cholerae strains in which the wild type gene was replaced by the mutant gene, which was confirmed by sequencing. The $\Delta 1785$ strain was designated $V$. cholerae strain SAM-3. A knockout mutant of VC1809 was constructed in N16961 as described above using primer pairs listed in Table 2. Complementation of RAM-1 and SAM-3 mutant strains were generated by creating pIntV2 and pVefA, by cloning intV2 (VC1758) and vefA (VC1785), respectively into the SacI/XbaI sites of the expression plasmid pBAD33 (New England Biolabs) using standard cloning protocol (Table 1 and 2).

\section{Results and Discussion}

VPI-2 excision rates under different growth conditions

It was previously shown that the four pathogenicity islands identified in $V$. cholerae N16961 can excise from chromosome 1 and form circular intermediates (CI) $[23,28]$. The excision of VPI-1 and VPI-2 occurs at very low levels suggesting that excision is tightly controlled, although it may also suggest that the excision event is inefficient, possibly due to poor expression of the regulatory genes, an altered regulatory circuit, or mutations that might occur in these sequences as the region become evolutionarily integrated into the host chromosome [23,28]. First, we quantified the excision levels of VPI-2 in cultures of $V$. cholerae N16961 grown for 12 hours in $\mathrm{LB}$ at $37^{\circ} \mathrm{C}$ (standard conditions) by measuring the presence of $a t t B$, the locus present on the chromosome after VPI-2 excises (Figure 1), and comparing it with the housekeeping gene $m d h$ using QPCR. We used $a t t B$ as a surrogate for VPI-2 excision measurements since the copy number of attP in the CI is minuscule compared to $a t t B$, which replicates along with the rest of the chromosome unlike excised VPI-2. We compared the presence of $a t t B$ with $m d h$ since all cells encode one functional copy of the latter. PCR products of $a t t B$ and $m d h$ were visually checked on an agarose gel and their melting temperature analyzed to ensure we had the correct PCR products. The reference gene was assayed both separately and in the same reaction. Both primer pairs used were tested by comparing the results obtained using previously quantified cloned copies of $m d h$ and $a t t B$ and gave comparable results. We found that $a t t B$ was present in 1 in every $1.6( \pm 0.2) \times 10^{6} \mathrm{~V}$. cholerae cells under optimal growth conditions.

Next, we measured the presence of attB from $V$. cholerae cells cultured under different conditions compared with the presence of $a t t B$ under our standard condition, growth for 12 hours at $37^{\circ} \mathrm{C}$. We determined that incubation time does not affect the excision levels of VPI-2 indicating that excision does not occur in a growth phase dependent manner (Figure 2). However, V. cholerae cultures grown at $25^{\circ} \mathrm{C}$ showed a 2-fold increase in the presence of the $a t t B$ site compared to cells grown at the optimum temperature $37^{\circ} \mathrm{C}$ (Figure 2). In addition, we found that nutrient limitation affected the excision level showing over a 5 -fold decrease in the presence of $a t t B$ when compared to the growth on LB at the same temperature (Figure 2). Furthermore, we found that sub-lethal UV-light irradiation of cell cultures compared to untreated cells, resulted in a significant increase in the level of excision of VPI-2, over 4-fold compared to untreated cells grown under the same conditions 


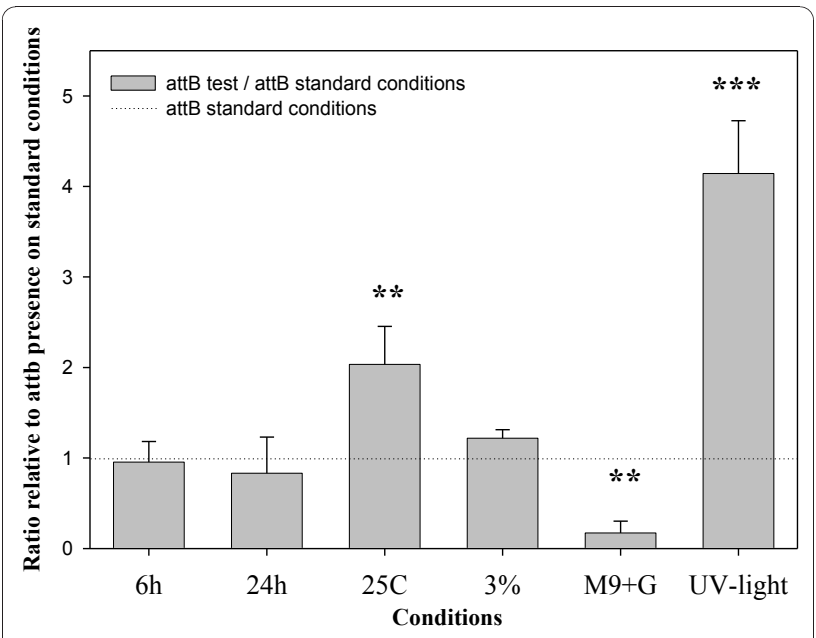

Figure 2 Detection of VPI-2 excision by using real time quantitative PCR (QPCR) of attB levels in cell cultures grown under different conditions. The $X$-axis specifies culture conditions: $6 \mathrm{~h}$, incubation time of $6 \mathrm{~h} ; 24 \mathrm{~h}$, incubation time of $24 \mathrm{~h} ; 25 \mathrm{C}$, incubation temperature of $25^{\circ} \mathrm{C} ; 3 \%$, the $L B$ broth contained $3 \%$ $\mathrm{NaCl} ; \mathrm{M} 9+\mathrm{G}$, cell grown on minimal media supplemented with glucose; UV-light, bacterial cultures were UV-light irradiated. The Y-axis represent the ratio of the attB presence in the cultures tested compared with cultures grown on standard conditions $12 \mathrm{~h}$ at $37^{\circ} \mathrm{C}$ in LB. Unpaired $t$-test was used in order to infer statistical significance for the differences in VPI-2 excision. ${ }^{* *}, \mathrm{p}<0.005$

**, $p<0.05$. Error bars indicate standard deviation. Each experiment was performed in triplicate a minimum of three times.

(Figure 2). Taken together, these data indicate that environmental factors can affect the induction of excision and circularization of VPI-2, which is probably the first step required for the horizontal transfer of the region. These results are consistent with what was previously shown for other mobile and integrative genetic elements as well as PAIs from E. coli, where excision occurs upon exposure to stress conditions such as sublethal UV-light irradiation $[53,55,56]$.

\section{VPI-2 encodes two novel recombination directionality factors}

Both the high pathogenicity island HPI from $Y$. pestis and ICE SXT from $V$. cholerae encode small accessory proteins called recombination directionality factors (RDFs) or excisionases (Xis) that are required for efficient excision of these elements $[29,41]$. In order to identify candidate RDFs within VPI-2 from $V$. cholerae N16961, we performed BLAST and PSI-BLAST searches on the $V$. cholerae N16961 genome using RDFs, the $V$. cholerae Xis protein (ABA87014) from SXT, the $Y$. pestis Hef protein (NP_405464) from HPI and E. coli K12 AlpA protein (AAA18418) from $\lambda$ phage as queries [57]. The most significant BLAST result in these searches was ORF VC0497, which is annotated as a transcriptional regulator, and is encoded within Vibrio

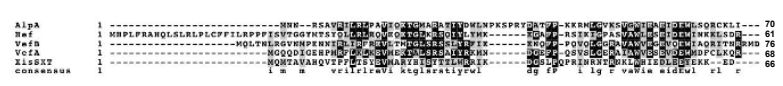

Figure 3 Alignments of VPI-2 RDFs VefA and VefB with other known RDFs: AlpA (AAA18418), Hef (NP_405464), Xis

(ABA87014). For both alignments black shading highlights residues shared by all the sequences and grey shading highlights residues shared by all but one sequences. The numbers on the right indicate the number of amino acids of the predicted protein.

Seventh Pandemic island-II (VSP-II). VSP-II also encodes a tyrosine recombinase integrase at ORF VC0516 (IntV3) [58]. ORFs VC1785 and VC1809 encoded within VPI-2 were the second and third most significant hits retrieved from these BLAST searches, which we termed VefA (for Vibrio excision factor A) and VefB, respectively (Figure 3). The VefA and VefB proteins share $46 \%$ amino acid identity/72\% similarity. VefA shares 37\% amino acid identities with AlpA, 46\% identity with Hef and $29 \%$ with Xis from the $V$. cholerae SXT element as was previously shown [53] (Figure 3). The vefB gene is located at the 3' end of VPI-2 at ORF VC1809 marking the end of the island, and vefA (VC1785) is adjacent to neuraminidase gene, nanH (VC1784) in the middle of the island (Figure 1A).

As shown in Figure 2, UV-light irradiation increased excision of VPI-2 over 4-fold. In order to investigate this further, we determined the effect of UV-light irradiation on the expression of int $V 2$, vefA and vefB in $V$. cholerae N16961 (Figure 4). We examined transcript levels of intV2, vefA and vefB in cells grown for $12 \mathrm{~h}$ in LB and in cells grown for $12 \mathrm{~h}$ in LB followed UV-light irradiation treatment. We found that all three genes showed negligible levels of transcription under standard optimum growth conditions but after UV-light treatment both int $V 2$ and vefA show a 10 -fold and $v e f B$ a 5 -fold increase in expression levels (Figure 4). These results indicate that UV-light induces expression of factors potentially involved in VPI-2 excision.

\section{IntV2 and VefA are essential for the excision of VPI-2}

To determine in more detail the role of intV2, vefA and $v e f B$ in VPI-2 excision, we created deletion mutations in each gene and measured excision levels of VPI-2 by determining attB levels in cells. In $V$. cholerae RAM-1, an intV2 mutant, we did not detect any VPI-2 attB products, demonstrating that intV2 is essential for excision as was previously shown (Figure 5) [23]. We complemented RAM-1 with a functional copy of int $V 2$ by transforming $V$. cholerae RAM-1 with pIntV2 creating strain SAM-1. In our SAM-1 strain, we found that excision of VPI-2 was restored in addition, attB levels were approximately four-fold higher than wild-type levels which is represented by the dotted broken horizontal 


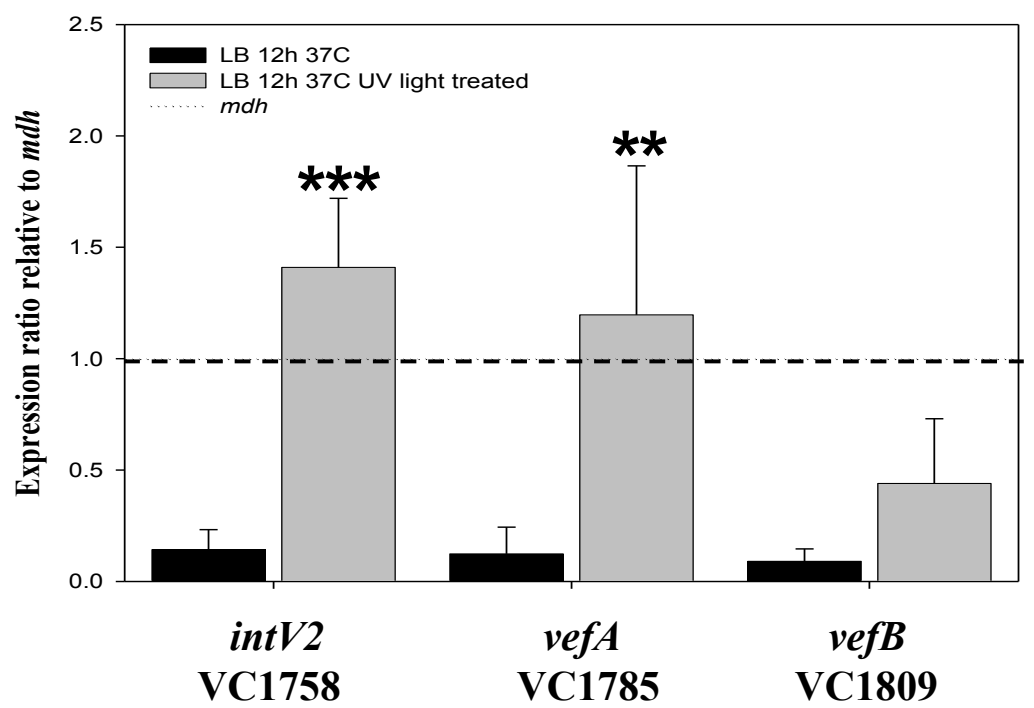

Figure 4 Expression of intV2 (VC1758), vefA, and vefB from cultures grown in standard (black bars) or UV-light irradiated cultures (grey bars). The $Y$-axis represents the expression ratio of the genes relative to the expression of mdh. Unpaired $t$-test was used in order to infer statistical significance for the differences in gene expression between cultures of $V$. cholerae $N 16961$ with or without UV-light treatment. ${ }^{*}$, $P<$ $0.05 ;{ }^{* *}, p<0.005$. Error bars indicate standard deviation. Each experiment was performed in triplicate a minimum of three times.

line in Figure 5. These data demonstrate that over expressing int $V 2$ ectopically induces excision of VPI-2. In our control experiments, transformation of either wild-type N16961 or RAM-1 with pBAD33 alone (strains SAM-11 and SAM-12 respectively) did not affect attB levels (data not shown).

qPCR-measured rates of excision for mutant and mutant-complement straints

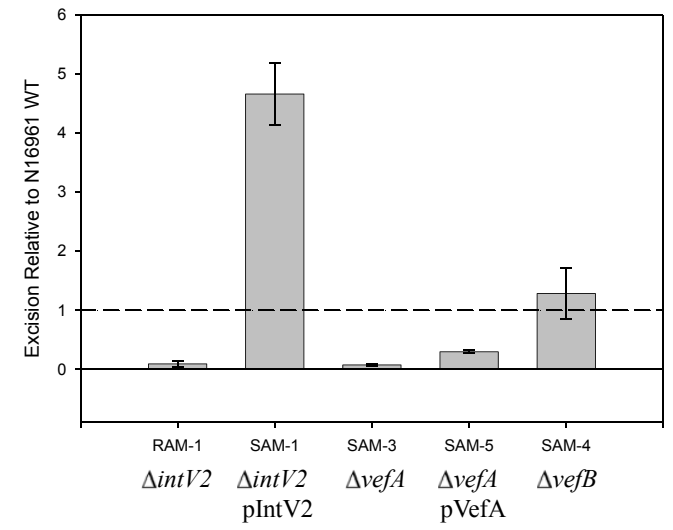

Figure 5 Excision levels of VPI-2 in mutant strains and strains complemented with intV2 (VC1758), and vefA (VC1785). Excision levels of $\triangle i n t V 2$ mutant (RAM-1), $\triangle i n t V 2$ mutant complemented (SAM-1), $\triangle$ vefA mutant (SAM-3), $\triangle$ vefA mutant complemented (SAM5), and $\triangle$ vefB mutant (SAM-4). Unpaired $t$-test was used in order to infer statistical significance for the differences in VPI-2 excision between $V$. cholerae N16961 and test strains. **, p < 0.05; ***, p < 0.005. Error bars indicate standard deviation. Each experiment was performed in triplicate a minimum of three times.
Next, we determined whether one, both, or neither of the putative RDFs uncovered by our bioinformatic analysis are required for VPI-2 excision. To do this, we constructed inframe deletion mutations in each gene to create mutant strain SAM-3 ( $\Delta v e f A)$ and SAM-4 ( $\Delta v e f B)$. The two mutant strains and the wild-type N16961 were each inoculated into LB and all three strains grew similarly indicating that the mutant constructs did not have any general growth defect (data not shown). We determined the attB levels using QPCR in strain SAM-3 compared to the wildtype strain grown under the same conditions. We found that no VPI-2 excision occurs in SAM-3 cells when compared with the wild type, indicating that a functional copy of vefA is essential for efficient excision of VPI-2 (Figure 5). We complemented SAM-3 with a functional copy of vefA (SAM-5) and measured attB levels in these cells with the wild type levels both under standard conditions, to find that some excision occurred, but it was less than in wild-type cells (Figure 5). In our vefB mutant strain (SAM-4), we found no difference in VPI-2 excision levels compared to wild-type grown under the same conditions, which demonstrates that $v e f B$ is not essential for excision (Figure 5). From these data it appears that vefA is the cognate RDF for VPI-2 excision. In our control experiments, transformation of SAM-3 with pBAD33 alone (resulting in strain SAM-13) did not affect attB levels (data not shown).

\section{Vibrio species island-encoded integrases with} corresponding RDFs

Given that our initial search for RDFs within one $V$. cholerae genome (strain N16961) yielded three 
putative RDFs (VC0497, VC1785, and VC1809), we decided to investigate further the occurrence of RDFs among Vibrio species whose genome sequence is available in the database. We performed BLAST searches against the 20 Vibrio species in the genome database, and we uncovered a total of 27 putative RDFs (Table 3). Next, we identified putative integrases within the genomes of the RDF homologues using BLAST search analysis by using IntV2 as a seed. For each of the RDFs identified among the 27 genomes encompassing 10 different Vibrio species (V. cholerae, $V$. coralliilyticus, $V$. furnissii, V. harveyi, V. parahaemolyticus, V. splendidus, V. vulnificus, Vibrio sp. Ex25, RC341, and MED222), we identified a corresponding integrase with greater than $40 \%$ amino acid identities to IntV2 (VC1758) (Table 3). We examined the gene context of

Table 3 Locus tags for integrases and corresponding RDFs identified in this study.

\begin{tabular}{|c|c|c|}
\hline & Integrases & RDFs \\
\hline Species Strain & Locus tag & Locus tag \\
\hline Vibrio cholerae N16961* & VC1758 & VC1785NC1809 \\
\hline $\begin{array}{l}\text { Vibrio cholerae TM 11079- } \\
80\end{array}$ & VIF_001175 & VIF_000799 \\
\hline Vibrio cholerae TMA21 & VCB_002798 & VCB_002857 \\
\hline Vibrio cholerae 12129(1) & VCG_002315 & VCG_002259 \\
\hline Vibrio cholerae V51 & VCV51_1204 & VCV51_0550 \\
\hline Vibrio cholerae 1587 & A55_1986 & A55_2025 \\
\hline Vibrio cholerae CT 5369-93 & VIH_002346 & VIH_002364 \\
\hline Vibrio cholerae RC385 & VCRC385_0574 & VCRC385_3603 \\
\hline Vibrio cholerae TMA 21 & VCB_000212 & VCB_000197 \\
\hline Vibrio cholerae MZO-3 & A51_B0496 & A51_B0476 \\
\hline Vibrio cholerae 12129(1) & VCG_003155 & VCG_003160 \\
\hline Vibrio cholerae N16961* & VC0516 & VC0497 \\
\hline Vibrio cholerae MZO-3 & A51_B0965 & A51_B0948 \\
\hline Vibrio vulnificus YJO16* & W2262 & W2261 \\
\hline Vibrio vulnificus YJ016* & W0817 & W0810 \\
\hline Vibrio vulnificus YJO16* & W0560 & W0515 \\
\hline Vibrio furnissii CIP 102972 & VFA_001916 & VFA_001914 \\
\hline Vibrio furnissii CIP 102972 & VFA_000464 & VFA_000468 \\
\hline $\begin{array}{l}\text { Vibrio coralliilyticus ATCC } \\
\text { BAA- } 450\end{array}$ & VIC_001980 & VIC_001987 \\
\hline Vibrio sp. Ex25 & VEA_004301 & VEA_004310 \\
\hline Vibrio sp. RC341 & VCJ_000330 & VCJ_000314 \\
\hline Vibrio sp. MED222 & MED222_15534 & MED222_15529 \\
\hline Vibrio splendidus $12 \mathrm{~B} 01$ & V12B01_04993 & V12B01_05053 \\
\hline $\begin{array}{l}\text { Vibrio parahaemolyticus } \\
\text { AQ3810 }\end{array}$ & A79_5467 & A79_5463 \\
\hline $\begin{array}{l}\text { Vibrio parahaemolyticus } \\
\text { K5030 }\end{array}$ & VparK_010100010115 & VparK_010100010135 \\
\hline $\begin{array}{l}\text { Vibrio parahaemolyticus } \\
\text { AQ3810 }\end{array}$ & A79_2546 & A79_2541 \\
\hline Vibrio harveyi HY01 & A1Q_2023 & A1Q_2003 \\
\hline
\end{tabular}

* indicates a genome that is completely annotated each RDF and integrase within each of the 20 strains to determine whether the RDF and integrase were located on the same region within a strain. From these analyses, we found that each of the 27 RDFs has a corresponding integrase within approximately $100 \mathrm{~kb}$ of each other (Table 3). It should be noted that from table 3, only three of the strains have been annotated completely and for many of the strains examined their ORF annotation numbering is not consecutive. Within $V$. cholerae, integrases and RDFs located in the same region of the genome in different strains had the same gene content indicating the same island is present in different strains. Among the different species, however, integrases and RDFs associated with the same insertion site did not have the same gene content indicating a novel island region in the different species (data not shown).

From our analysis, no RDF was identified within the VPI-1 or the VSP-I regions in N16961 or within homologous regions in the other 27 sequenced strains of $V$. cholerae in the database. Both the VPI-1 and VSP-I regions have been shown to excise from their chromosome location, and VPI-1 encodes a tyrosine recombinase with homology to IntV2, thus they may therefore use an alternative mechanism of excision or perhaps coopt an RDF from another region on the genome. Overall our data indicates that the presence of both an integrase and a cognate RDF pairing is a relatively conserved feature but not an essential one.

\section{Conclusions}

In this study, we analyzed the excision dynamics of VPI-2 encoded within $V$. cholerae N16961. Our results indicate that excision is controlled by at least two conserved factors within the island, an integrase encoded by int $V 2$ and an RDF encoded by vefA, whose expression is induced by environmental stimuli similar to other MIGEs such as prophages, ICEs and integrons. We identified two putative RDFs and found that of the two we identified, only one VefA is essential for the efficient excision of VPI-2. We determined the occurrence of RDFs among the genomes of sequenced Vibrio species and found 27 putative RDFs that also had a homologue of IntV2 associated with it, which suggests that requirement for both an RDF and a corresponding integrase is a relatively common feature.

\section{Acknowledgements}

This research was supported by National Science Foundation CAREER award DEB-0844409 to E.F.B.

The authors declare no conflicts of interest.

\section{Author details}

${ }^{1}$ Department of Biological Sciences, University of Delaware, Newark, DE

19716 USA. ${ }^{2}$ Dartmouth Medical School, Dept. of Microbiology and Immunology, Vail Bldg. Rm 106, Hanover, NH 03755. 


\section{Authors' contributions}

EFB designed the research; SA-M and MGN performed the research; SA-M, MGN and EFB analyzed data; SA-M, MGN and EFB wrote the paper.

Received: 19 August 2010 Accepted: 30 November 2010

Published: 30 November 2010

\section{References}

1. Faruque SM, Sack DA, Sack RB, Colwell RR, Takeda Y, Nair GB: Emergence and evolution of Vibrio cholerae 0139. Proc Natl Acad Sci USA 2003, 100(3):1304-1309.

2. Faruque SM, Chowdhury N, Kamruzzaman M, Dziejman M, Rahman MH, Sack DA, Nair GB, Mekalanos JJ: Genetic diversity and virulence potential of environmental Vibrio cholerae population in a cholera-endemic area. Proc Natl Acad Sci USA 2004, 101(7):2123-2128.

3. Burrus V, Quezada-Calvillo R, Marrero J, Waldor M: SXT-related integrating conjugative element in New World Vibrio cholerae. Appl Environ Microbiol 2006, 72:3054-3057.

4. Nusrin S, Gil Al, Bhuiyan NA, Safa A, Asakura M, Lanata CF, Hall E, Miranda H, Huapaya B, Vargas GC, et al: Peruvian Vibrio cholerae O1 El Tor strains possess a distinct region in the Vibrio seventh pandemic island-II that differentiates them from the prototype seventh pandemic El Tor strains. J Med Microbiol 2009, 58:342-354.

5. Tay C, Reeves P, Lan R: Importation of the major pilin TcpA gene and frequent recombination drive the divergence of the Vibrio pathogenicity island in Vibrio cholerae. FEMS Microbiol Lett 2008, 289:210-218,

6. Ghosh R, Nair GB, Tang L, Morris JG, Sharma NC, Ballal M, Garg P, Ramamurthy T, Stine OC: Epidemiological study of Vibrio cholerae using variable number of tandem repeats. FEMS Microbiol Lett 2008, 288(2):196-201.

7. Gonzalez-Fraga S, Pichel M, Binsztein N, Johnson JA, Morris JG Jr, Stine OC: Lateral gene transfer of $\mathrm{O} 1$ serogroup encoding genes of Vibrio cholerae. FEMS Microbiol Lett 2008, 286(1):32-38.

8. Chun J, Grim CJ, Hasan NA, Lee JH, Choi SY, Haley BJ, Taviani E, Jeon YS, Kim DW, Lee JH, et al: Comparative genomics reveals mechanism for short-term and long-term clonal transitions in pandemic Vibrio cholerae. Proc Natl Acad Sci USA 2009, 106(36):15442-1547.

9. Grim CJ, Hasan NA, Taviani E, Haley B, Chun J, Brettin TS, Bruce DC, Detter JC, Han CS, Chertkov O, et al: Genome sequence of hybrid Vibrio cholerae $01 \mathrm{MJ}-1236, \mathrm{~B}-33$, and CIRS101 and comparative genomics with V. cholerae. J Bacteriol 2010, 192(13):3524-3533.

10. Lam C, Octavia S, Reeves P, Wang L, Lan R: Evolution of seventh cholera pandemic and origin of 1991 epidemic, Latin America. Emerg Infect Dis 2010, 16:1130-1132.

11. Morita M, Ohnishi M, Arakawa E, Yamamoto S, Nair GB, Matsushita S, Yokoyama K, Kai A, Seto K, Watanabe H, et al: Emergence and genetic diversity of El Tor Vibrio cholerae $\mathrm{O} 1$ that possess classical biotype ctxB among travel-associated cases of cholera in Japan. J Med Microbiol 2010, 59(Pt 6):708-712

12. Safa A, Nair GB, Kong RY: Evolution of new variants of Vibrio cholerae 01 . Trends Microbiol 2010, 18(1):46-54.

13. De SN: Enterotoxicity of bacteria-free culture-filtrate of Vibrio cholerae. Nature 1959, 183(4674):1533-1534.

14. Waldor MK, Mekalanos Jj: Lysogenic conversion by a filamentous phage encoding cholera toxin. Science 1996, 272(5270):1910-1914.

15. Galen JE, Ketley JM, Fasano A, Richardson SH, Wasserman SS, Kaper JB: Role of Vibrio cholerae neuraminidase in the function of cholera toxin. Infect Immun 1992, 60(2):406-415.

16. Jermyn WS, Boyd EF: Molecular evolution of Vibrio pathogenicity island-2 (VPI-2): mosaic structure among Vibrio cholerae and Vibrio mimicus natural isolates. Microbiology 2005, 151(Pt 1):311-322.

17. Dziejman $\mathrm{M}$, Balon E, Boyd D, Fraser CM, Heidelberg JF, Mekalanos JJ: Comparative genomic analysis of Vibrio cholerae: genes that correlate with cholera endemic and pandemic disease. Proc Natl Acad Sci USA 2002, 99(3):1556-1561.

18. Jermyn WS, Boyd EF: Characterization of a novel Vibrio pathogenicity island (VPI-2) encoding neuraminidase (nanH) among toxigenic Vibrio cholerae isolates. Microbiology 2002, 148(Pt 11):3681-3693.

19. Almagro-Moreno S, Boyd EF: Sialic Acid Catabolism Confers a Competitive Advantage to Pathogenic Vibrio cholerae in the Mouse Intestine. Infect Immun 2009, 77(9):3807-3816.
20. Almagro-Moreno S, Boyd EF: Insights into the evolution of sialic acid catabolism among bacteria. BMC Evol Biol 2009, 9(1):118.

21. Dziejman M, Serruto D, Tam VC, Sturtevant D, Diraphat P, Faruque SM, Rahman MH, Heidelberg JF, Decker J, Li L, et al: Genomic characterization of non-01, non-0139 Vibrio cholerae reveals genes for a type III secretion system. Proc Natl Acad Sci USA 2005, 102(9):3465-3470.

22. Chen Y, Johnson JA, Pusch GD, Morris JG Jr, Stine OC: The genome of non-01 Vibrio cholerae NRT36 S demonstrates the presence of pathogenic mechanisms that are distinct from those of 01 Vibrio cholerae. Infect Immun 2007, 75(5):2645-2647.

23. Murphy RA, Boyd EF: Three pathogenicity islands of Vibrio cholerae can excise from the chromosome and form circular intermediates. J Bacteriol 2008, 190(2):636-647.

24. Alam A, Tam V, Hamilton E, Dziejman M: vttRA and vttRB Encode ToxR family proteins that mediate bile-induced expression of type three secretion system genes in a non-01/non-0139 Vibrio cholerae strain. Infect Immun 2010, 78(6):2554-2570.

25. Tam VC, Serruto D, Dziejman M, Brieher W, Mekalanos JJ: A type III secretion system in Vibrio cholerae translocates a formin/spire hybridlike actin nucleator to promote intestinal colonization. Cell Host Microbe 2007, 1(2):95-107.

26. Karaolis DK, Johnson JA, Bailey CC, Boedeker EC, Kaper JB, Reeves PR: A Vibrio cholerae pathogenicity island associated with epidemic and pandemic strains. Proc Natl Acad Sci USA 1998, 95(6):3134-3139.

27. Taylor RK, Miller VL, Furlong DB, Mekalanos JJ: Use of phoA gene fusions to identify a pilus colonization factor coordinately regulated with cholera toxin. Proc Natl Acad Sci USA 1987, 84(9):2833-2837.

28. Rajanna C, Wang J, Zhang D, Xu Z, Ali A, Hou YM, Karaolis DK: The vibrio pathogenicity island of epidemic Vibrio cholerae forms precise extrachromosomal circular excision products. J Bacteriol 2003, 185(23):6893-6901.

29. Buchrieser C, Brosch R, Bach S, Guiyoule A, Carniel E: The highpathogenicity island of Yersinia pseudotuberculosis can be inserted into any of the three chromosomal asn tRNA genes. Mol Microbiol 1998, 30(5):965-978.

30. Buchrieser C, Prentice M, Carniel E: The 102-kilobase unstable region of Yersinia pestis comprises a high-pathogenicity island linked to a pigmentation segment which undergoes internal rearrangement. J Bacteriol 1998, 180(9):2321-2329.

31. Hochhut B, Wilde C, Balling G, Middendorf B, Dobrindt U, Brzuszkiewicz E, Gottschalk G, Carniel E, Hacker J: Role of pathogenicity island-associated integrases in the genome plasticity of uropathogenic Escherichia coli strain 536. Mol Microbiol 2006, 61(3):584-595.

32. Lesic B, Bach S, Ghigo JM, Dobrindt U, Hacker J, Carniel E: Excision of the high-pathogenicity island of Yersinia pseudotuberculosis requires the combined actions of its cognate integrase and Hef, a new recombination directionality factor. Mol Microbiol 2004, 52(5):1337-1348.

33. Middendorf B, Hochhut B, Leipold K, Dobrindt U, Blum-Oehler G, Hacker J: Instability of pathogenicity islands in uropathogenic Escherichia coli 536. J Bacteriol 2004, 186(10):3086-3096.

34. Sakellaris H, Luck SN, Al-Hasani K, Rajakumar K, Turner SA, Adler B: Regulated site-specific recombination of the she pathogenicity island of Shigella flexneri. Mol Microbiol 2004, 52(5):1329-1336.

35. Schubert S, Dufke S, Sorsa J, Heesemann J: A novel integrative and conjugative element (ICE) of Escherichia coli: the putative progenitor of the Yersinia high-pathogenicity island. Mol Microbiol 2004, 51(3):837-848.

36. Wilde C, Mazel D, Hochhut B, Middendorf B, Le Roux F, Carniel E, Dobrindt $U$, Hacker J: Delineation of the recombination sites necessary for integration of pathogenicity islands II and III into the Escherichia coli 536 chromosome. Mol Microbiol 2008, 68(1):139-151.

37. Blum G, Ott M, Lischewski A, Ritter A, Imrich H, Tschape H, Hacker J: Excision of large DNA regions termed pathogenicity islands from tRNAspecific loci in the chromosome of an Escherichia coli wild-type pathogen. Infect Immun 1994, 62(2):606-614.

38. Hacker J, Blum-Oehler G, Muhldorfer I, Tschape H: Pathogenicity islands of virulent bacteria: structure, function and impact on microbial evolution. Mol Microbiol 1997, 23(6):1089-1097.

39. Dobrindt U, Blum-Oehler G, Nagy G, Schneider G, Johann A, Gottschalk G, Hacker J: Genetic structure and distribution of four pathogenicity islands (PAI I(536) to PAI IV(536)) of uropathogenic Escherichia coli strain 536. Infect Immun 2002, 70(11):6365-6372. 
40. Lewis JA, Hatfull GF: Control of directionality in integrase-mediated recombination: examination of recombination directionality factors (RDFs) including Xis and Cox proteins. Nucleic Acids Res 2001, 29(11):2205-2216.

41. Burrus V, Waldor MK: Control of SXT integration and excision. J Bacteriol 2003, 185(17):5045-5054.

42. Luck SN, Turner SA, Rajakumar K, Adler B, Sakellaris H: Excision of the Shigella resistance locus pathogenicity island in Shigella flexneri is stimulated by a member of a new subgroup of recombination directionality factors. J Bacteriol 2004, 186(16):5551-5554.

43. Bushman W, Thompson JF, Vargas L, Landy A: Control of directionality in lambda site specific recombination. Science 1985, 230(4728):906-911.

44. Kim S, Landy A: Lambda Int protein bridges between higher order complexes at two distant chromosomal loci attL and attR. Science 1992, 256(5054):198-203.

45. Kim S, Moitoso de Vargas L, Nunes-Duby SE, Landy A: Mapping of a higher order protein-DNA complex: two kinds of long-range interactions in lambda attL. Cell 1990, 63(4):773-781.

46. Franz B, Landy A: The Holliday junction intermediates of lambda integrative and excisive recombination respond differently to the bending proteins integration host factor and excisionase. Embo J 1995, 14(2):397-406.

47. Moitoso de Vargas L, Landy A: A switch in the formation of alternative DNA loops modulates lambda site-specific recombination. Proc Natl Acad Sci USA 1991, 88(2):588-592.

48. Sam MD, Cascio D, Johnson RC, Clubb RT: Crystal structure of the excisionase-DNA complex from bacteriophage lambda. J Mol Biol 2004, 338(2):229-240.

49. Bertani G: Lysogeny at mid-twentieth century: P1, P2, and other experimental systems. J Bacteriol 2004, 186:595-600.

50. Pfaffl MW: A new mathematical model for relative quantification in realtime RT-PCR. Nucleic Acids Res 2001, 29(9):e45.

51. Altschul SF, Madden TL, Schaffer AA, Zhang J, Zhang Z, Miller W, Lipman DJ: Gapped BLAST and PSI-BLAST: a new generation of protein database search programs. Nucleic Acids Res 1997, 25(17):3389-3402.

52. Larkin MA, Blackshields G, Brown NP, Chenna R, McGettigan PA, McWilliam H, Valentin F, Wallace IM, Wilm A, Lopez R, et al: Clustal W and Clustal X version 2.0. Bioinformatics 2007, 23(21):2947-2948.

53. Quinones M, Kimsey HH, Waldor MK: LexA cleavage is required for CTX prophage induction. Mol Cell 2005, 17(2):291-300.

54. Horton RM, Hunt HD, Ho SN, Pullen JK, Pease LR: Engineering hybrid genes without the use of restriction enzymes: gene splicing by overlap extension. Gene 1989, 77(1):61-68.

55. Beaber JW, Hochhut B, Waldor MK: SOS response promotes horizontal dissemination of antibiotic resistance genes. Nature 2004, 427(6969):72-74.

56. Guerin E, Cambray G, Sanchez-Alberola N, Campoy S, Erill I, Da Re S, Gonzalez-Zorn B, Barbe J, Ploy MC, Mazel D: The SOS response controls integron recombination. Science 2009, 324(5930):1034

57. Heidelberg JF, Eisen JA, Nelson WC, Clayton RA, Gwinn ML, Dodson RJ, Haft DH, Hickey EK, Peterson JD, Umayam L, et al: DNA sequence of both chromosomes of the cholera pathogen Vibrio cholerae. Nature 2000, 406(6795):477-483.

58. O'Shea YA, Finnan S, Reen FJ, Morrissey JP, O'Gara F, Boyd EF: The Vibrio seventh pandemic island-II is a $26.9 \mathrm{~kb}$ genomic island present in Vibrio cholerae El Tor and 0139 serogroup isolates that shows homology to a $43.4 \mathrm{~kb}$ genomic island in V. vulnificus. Microbiology 2004, 150(Pt 12):4053-4063.

59. Philippe N, Alcaraz JP, Coursange E, Geiselmann J, Schneider D: Improvement of pCVD442, a suicide plasmid for gene allele exchange in bacteria. Plasmid 2004, 51(3):246-255.

60. Guzman LM, Belin D, Carson MJ, Beckwith J: Tight regulation, modulation, and high-level expression by vectors containing the arabinose PBAD promoter. J Bacteriol 1995, 177(14):4121-4130.

\section{Submit your next manuscript to BioMed Central and take full advantage of:}

- Convenient online submission

- Thorough peer review

- No space constraints or color figure charges

- Immediate publication on acceptance

- Inclusion in PubMed, CAS, Scopus and Google Scholar

- Research which is freely available for redistribution

Submit your manuscript at www.biomedcentral.com/submit 